\title{
On the Research of the Smart Grid Load Forecasting Cloud Platform Architecture
}

\author{
Mingyue Zhai ${ }^{1}$ \\ 'School of Computer and Information Engineering \\ Guangdong University Of Petrochemical Technology, Maoming, China
}

Keywords: Load forecasting; Cloud platform; Load forecasting method; Smart grid; Smart meter

\begin{abstract}
Load forecasting is making calculations or predictions of a future event or condition based on analysis or study of historical data, events or observations. With the development of smart grid, smart meter is also increasing largely, which can generate metering data per 15 mins, and there comes a huge amount of metering data. Thus such huge amount of data leads to some challenges in data processing. This paper introduces the meaning of studying load forecasting, and recommends current relevant research. Then, the cloud platform architecture, the key technologies based cloud and current relevant research for load forecasting based on cloud in smart grid is discussed. Finally it introduces the related load forecasting services based on the cloud platform architecture in smart grid. After all, there is a conclusion of this paper.
\end{abstract}

\section{Introduction}

In the past three decades, many experts and scholars at domestic and abroad have done lots of research works on the load forecasting methods and made many achievements. The development of prediction methods has gone through four stages: (1) traditional historical statistics prediction represented by linear regression, moving average; (2) time series prediction proposed by Box-Jenkins (including AR, MA and ARMA); (3) gray model and combination prediction; (4) intelligent prediction including the learning algorithm based on neural networks and support vector machine and the optimization algorithm based on particle swarm algorithm and genetic algorithm.

With the rapid development of smart grid, smart meters are used widely with generating vast amounts of data, using these data fully can help to realize the power load analysis and forecasting. This requires researchers to find an appropriate way to forecast the power loading, and ensure the grid to operate safely and stably.

In this paper, firstly, a few methods of load forecasting are introduced, and then cloud computing based smart meter analysis and forecasting methods and framework are discussed. After all, there is a conclusion of this paper.

\section{The method of forecasting power load}

The analysis and forecasting for power load is an important part in power system planning and operation, which can ensure the operation reliability of the power system. The results of prediction are used to determine the power demand and the grid capacity within an area in the future. According to the different forecast period scale, it has long-term forecast, medium-term forecast, short-term forecasting and ultra-short-term forecasting, and the corresponding is annual forecasting, monthly forecasting, daily forecasting and hourly forecasting [1].

With the emergence of smart meters, there is a lot of literature research on the smart meter data for load analysis. The literature [2] uses the artificial neural networks combining with the weather data and historical data of smart meters to forecast the load in a few days later. For the defects of traditional load forecasting model, [3] proposes a gray short-term load forecasting method based on meteorological factor correction, which identifies the abnormal problem in the historical day temperature sequence and corrects it, and improves the accuracy of prediction . Jo Li et al. [4] applies the thinking of weight scale method to carry out the medium-term and long-term forecasting for power load. A short-term load forecasting method for users is carried out in [5] counting the 
relationship of electricity consumption among time series, which is based on machine learning techniques considering the relevance of families. Long-term load forecasting is determined by energy consumption, income, the rate of population growth and economic, [6] and considers these factors together to carry out long-term forecasting for the peak load in the region of Turkey by using the artificial neural networks and artificial neural fuzzy inference system. [7] proposes a gray neural network model based on the improved genetic optimization algorithm to forecast short-term load in the condition of real-time pricing, and uses genetic algorithm to optimize network so that improve the prediction accuracy. [8] studies that evidence theory of NALM and DS is used to detect the activity patterns of users.

The literature [9] focuses on studying the behavior of smart power under the smart grid based on the analysis of smart grid. The literature [10] discusses the experience of smart meter data based on the characterization, modeling and data analysis based model. [11] introduces that by using clustering algorithm to deals the smart meter data realizes the resolution of load. [12] shows the subspace method to certificate the household appliances based on the smart meter data. [13] researches on the applications of the smart meter data in the user classification and load profiling. The literature [14] introduces the artificial neural networks to forecast the electricity load, for the real-time data collected by the smart meter with high frequency and fast speed, proposed a BP neural network method to analyze and forecast the trend of user behavior in each period of the future. Adjust the price timely based on predicting the results can help save the cost of grid operation, reduce the maximum power load and improve the operational efficiency of the grid.

In addition, the analysis and forecast for the smart grid load is premises to provide grid state security monitoring and fault analysis. In the estimation for the distribution network state, the analysis information of the trend in the distribution network side is usually very inaccurate, mainly because the information is obtained by processing network models, attached estimates and the measured information in the high pressure side of substation together. By adding nodes on the side of the user will obtain more accurate information about the load and power loss so that avoid the overload of electrical equipment and the deterioration of power quality. Through integrating a large number of measurement data, it can estimate the unknown status and calibrate the accuracy of measurement data [7]. The literature [15] describes a method to predict the distribution of off-power by using the smart meter data. The literature [16] infers the state of low-voltage distribution network based on the smart meter data. The literature [17], based on the actual three-phase measurement information of smart meters, establishes a three-phase four-wire distribution network exponentially weighted least squares estimation robust model.

\section{Smart grid load forecastING based on cloud platform architecture}

Cloud computing is a virtualized, dynamic and scalable resources using virtualization technology, clustering applications, grid computing, distributed computing, distributed storage and parallel computing and so on, and provides reliable data storage, convenient and efficient network services, and powerful data processing model for users to achieve unified management and dynamic allocation of resources. With the development of smart grid, it will have a huge amount of data. It is very suit for smart grid with the characteristics of "demand-use, adaptable", therefore, according to the basic characteristics of the smart grid, relationship between smart grid and load forecasting, using the cloud platform architecture to achieve the storages of large data, analysis and forecasting is significance.

Smart grid load forecasting cloud is a private cloud for load analysis, forecasting, management and coordination of the results. Load forecasting cloud mechanism is the key to forecast the load for the power private cloud, it reflects on the process of handling power cloud resources. Load forecasting cloud mechanism need base on load forecasting cloud platform to achieve the optimized integration of heterogeneous resources, the automated management of infrastructure resources, the efficient management of massive grid data, the rapid calculation and analysis of power system. Therefore it is necessary to analyze the smart grid cloud platform architecture.

The Hadoop [18-22] platform is an open source, and its unique advantages can service the cloud computing of smart grid. To overcome the problems that the data can't be shared, and the data 
processing usually costs too much times, we use the Hadoop to deploy the cloud level. The core part consists of the Hadoop Common, MapReduce, HBase, HDFS and Zoo Keeper [23], and Figure 1 shows the cloud platform based on the architecture of Hadoop for smart grid load forecasting.

According to the important parts of Hadoop, load forecasting cloud platform based on Hadoop architecture involves in key technologies: virtualization technology that make physical resources logic so that the operating system can be shared, parallel programming model technology (MapReduce) that by using in the field of power system information collection can achieve parallel computing for large-scale datasets, the distribution and storage technology of mass data (GFS, HDFS) that by adapting the decentralized deployment strategy improves the balanced level of read and write, massive data management technology (HBase, BigTable) that supports for the processing of data, and the service-oriented SOA architecture that package the cloud computing. Smart grid load forecasting will using these technologies based on the Hadoop architecture to achieve the collection, storage, processing, analysis of the data, and the optimization of related prediction algorithm for the massive high dimensional load.

In recent years, many researchers do related research on cloud computing in the application of smart grid load forecasting. Literature [23], according to the basic characteristics of the smart grid, analyses the relationship of smart grid and load forecasting. It introduces power private cloud based on virtualization technology, and deployed the entire smart grid load forecasting cloud platform by using Hadoop infrastructure and virtualization technology, parallel programming model technology, the distribution and storage technology for mass data, management technology for mass data, service-oriented framework (SOA), etc. to form the main framework and achieve a comprehensive data sharing.

\section{Load forecasting service}

With the development of smart grid, not only the load forecast technology should be developed, we should also pay attention to the load forecast service. The operation of the smart devices leads to a novel scene with the of forecast objects diversification, forecast processing fine and the forecast system service. All kinds of intelligent load forecasting will turn into four kinds of important smart grid load services rendered to the users, i.e. LAaaS (Load Analysis as a Service), LFaaS (Load Forecasting as a Service), LMaaS (LoadManagement as a Service) and COaaS (Coordination Optimization as a Service). Users could access these services anytime and anywhere.

Basic load analysis service LAaaS. Load forecasting is based on load analysis, which is also the foundation of grid planning and optimizing the structure of power. Generally the characteristics of the load analysis.

Taking the air conditioning load characteristic analysis as an example, the air conditioning load can be divided into summer cooling load and winter heating load. Analysis of air conditioning load should consider the characteristics of seasonal time series. Estimation method is usually used in the extraction of air conditioning load, which considers whether the shape of the curve has changed when the electricity is used orderly (if it does, consider reduction firstly). Then, use the basic load method to extract air conditioning load. We can restore curves with (1), formula (2) and (3) are used to extract air conditioning load, in which the $\mathrm{P}_{i}$ is corrected value of the $i$-th load, $P_{c i}$ and $P_{o i}$ are the $i$-th load value of reference curve and object curve respectively, $P_{c \min }$ and $P_{o \min }$ are the minimum of reference curve and object curve respectively, $\mathrm{i}=1,2,3, \ldots, 96$, and $T$ refers to corrected coefficient.

Based on (2) and (3), we can roughly obtain daily maximum cooling load in summer and daily maximum heating load in winter, which are respectively related to the maximum temperature of summer and minimum temperature of winter. From the relationship between them, the change of air condition load is obtained after the temperature is changed. Based on (2) and (3), we can obtain daily air condition load curve, but sometimes the load curve will be negative. On one hand, air conditioning is not used widely in several months, central air conditioning load is not obvious, and the main is dispersed residents air conditioning load. On the other hand, it may is the estimation error in the above formula, because the error may be caused by the method of curve reduction and basic load calculation. In smart grid, more intelligent method of extracting air conditioning load should be 
researched and analyze the influence that air conditioning load on load forecasting are required. Otherwise, intelligent equipment of collecting air conditioning load is also needed.

Load forecasting service based on load analysis - LfaaS. In the cloud computing platform, load forecasting service will be more diverse, and load analysis will be more sophisticated and scientific. Load analysis is the premise of load forecast. By analyzing the load characteristics of different objects, the data processing and sample selection are carried out reliably, which lays the foundation for the load forecasting. Then, load analysis can get the main factors that affect the load curve and load index. These factors are important sources of load forecasting and correction model parameters. Therefore, the research method based on load analysis is more practical. For example, load analysis is basic idea in many previous studies, such as the prediction method based on load curve coefficient, ultra short term load forecasting based on load derivation, estimate the air conditioning load by using the relationship between summer cooling load and winter heating load and weather.
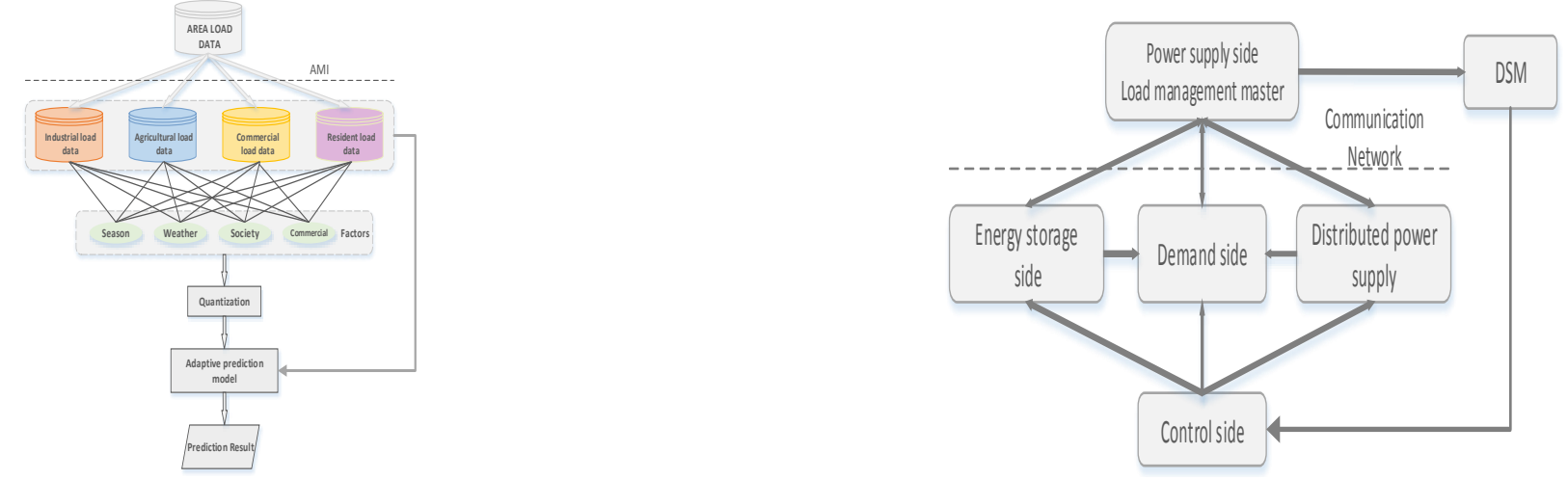

Fig. 1. Load forecasting service SaaS technology frame Fig.2. Load management service LMaaS technical framework for DSM

In order to predict profession load, smart devices will collect various professions (industry, agriculture, light industry, service industry, residential, commercial, etc.) data, considering different professions and different influencing factors. Each factor is quantified and is reflected in the forecasting model input. At the same time, the forecast result is also provided as a reference to the load management and the order use of electricity sector (see Fig. 2).

Load management service for power demand side management - LmaaS. Load management is an important part of Demand Side Management (DSM). Taking economic, administrative and technical measures as mainly means, intelligent terminal equipment as the carrier, safe economy for the operation of the power grid as a criterion, it can improve load curve, user specification behavior and forma rational consumption pattern, and it lays the foundation of load analysis and forecasting work. Load management for the DSM is superior to the traditional load control or management, which needs the cooperation of supplier (EDF) and consumer (user). It is conducive for load forecasting to make full use of the abundant consuming information, realize the interaction with users, find the shift peak potential of user side, and improve the smooth degree of the load forecasting curve.

\section{Conclusions}

According to the initial use of cloud computing in power system, cloud computing can solve several serious problems in current power system. It is proved that the cloud computing, smart grid, load forecasting can be closely combined. Therefore, it is of practical significance to study the smart grid load forecasting based on cloud computing. The combination of cloud computing and power load forecasting is just budding. More study of actual problem for the development of the power system load forecasting platform based on cloud computing are required. Future research should pay close attention to the current development needs for the load forecasting, and carefully study the new load forecasting technology at domestic and abroad. Also, we should focus on the research of distributed energy generation output forecasting, high-speed electrified railway power load forecasting, electricity load forecasting of typical large users in smart grid. 
This paper considered the load forecasting method and cloud services platform in smart grid as a keynote, the meaning of power load analysis, forecasting and modeling method are summarized firstly. Then, the methods of load forecasting in power grid are introduced in detail. Finally, the smart grid load forecasting cloud platform architecture, the key technologies based on cloud architecture, current relevant research based on cloud computing are introduced. And then especially the cloud computing information service layer of four load services are described, and corresponding technical frameworks are provided at last.

\section{References}

[1] Yadong Wang, GaoYan, JinFeng. 2015. Smart meter data analysis and applications. Information Technology, 2(18): 64-67.

[2] Asare-Bediako B, Kling W L, Ribeiro P F. 2013. Day-ahead residential load forecasting with artificial neural networks using smart meter data. Power Tech (POWERTECH), 2013 IEEE Grenoble. pp: 1-6.

[3] Ruimei Jiao, Cenjun Sun, Biying Lin, et al. 2013. Gray short-term load forecasting model based on meteorological information factor correction. Power Grid Technology, 37(3): 720-725.

[4] Li Zuo, Buxiang Zhou, LiJun, et al. 2013. Consider long-term load forecasting integrated model of the weight scale method. Journal of Electric Power System and Automation, 25(5): 96-99.

[5] Humeau S, Wijaya T K, Vasirani M, et al. 2013. Electricity load forecasting for residential customers: Exploiting aggregation and correlation between households. Sustainable Internet and ICT for Sustainability (SustainIT 2013), IEEE, pp: 1-6.

[6] Cetinkaya N. 2013. Long-term Electrical load forecasting based on economic and demographic data for Turkey. Computational Intelligence and Informatics (CINTI), 2013 IEEE 14th International Symposium on. IEEE, pp: 219-223.

[7] Shaoyun Ge, Ousha Jia, Liu Hong. 2012. Short-term load forecasting in the real-time pricing conditions based on genetic Grey Neural Network Model. Power Grid Technology, 36(1): 224-229.

[8] Jing Liao, Stankovic L., Stankovic V. 2014. Detectijng Household Activity Patterns from Smart Meter Data. Intelligent Environments 2014.

[9] Peifeng Du. 2014. Based on smart power analysis of the smart grid. Consumer Electronics, (22): 23-25.

[10] Sean Barker, Sandeep Kalra, David Irwin, Prashant Shenoy. 2014. Empirical characterization, modeling,and analysis of smart meter data. Areas in Communications, 32(7): 1312-1326.

[11] Vitaly Ford, Dr. Ambareen Siraj. 2013. Clustering of smart meter data for disaggregation. Global Conference on Signal and Information Processing (GlobalSIP), pp: 507-510.

[12] Dinesh, H.G.C.P., Nettasinghe, D.B.W., Godaliyadda, G.M.R.I. et al. A subspace signature based approach for residential appliances identification using less informative and low resolution smart meter data.

[13] Gheorghe Grigoras, Ovidiu Ivanov, and Mihai Gavrilas. 2014. Customer Classification and Load Profiling using Data from Smart Meters, 12: 25-27.

[14] Jianbo Zheng, Wang Fang. 2010. Smart Meter Data analysis and forecasting based on BP neural network. Power Grid Technology, 34(4): 17-19.

[15] Ken Kuroda, Ryuichi Yokoyama, Daisuke Kobayashi. 2014. An approach to outage location prediction utilizing Smart Metering Data. Computer Society, 8: 61-66.

[16] Qipeng Chen, Dritan Kaleshi, Zhong Fan. 2013. Inferring low voltage transformer state using only Smart Metering Data. Innovative Smart Grid Technologies Europe (ISGT Europe), October 6-9, 2013.

[17] YanWei, DuanLei, Huanyan Yang, ZhaoXia, YuJuan. 2015. Three-phase four-wire distribution network robust estimation based smart meter measurements. China Electrical Engineering, 1(35): 60-66. 